\title{
Image processing for the non-destructive characterization of porous media. Application to limestones and trabecular bones
}

- Ahmad Almhdie ${ }^{\text {ac }}$

- Olivier Rozenbaum ${ }^{\mathrm{a}}$

- Eric Lespessailles

- Rachid Jennane ${ }^{c}$

- $\quad{ }^{\text {a }}$ ISTO, UMR 7327, University of Orleans, 1A, rue de la Férollerie, 45071 Orléans, France

- ${ }^{\mathrm{b}}$ Univ. Orléans, I3MTO Laboratory, EA 4708, Hospital of Orleans, 1 rue Porte Madeleine, F-45032 Orléans, France

- ${ }^{\mathrm{c}}$ Univ. Orléans, PRISME Laboratory, EA 4229, 12 rue de Blois, BP 6744, F-45067 Orléans, France

\begin{abstract}
Different image processing techniques have recently been investigated for the characterization of complex porous media, such as bones, stones and soils. Among these techniques, 3D thinning algorithms are generally used to extract a one-voxel-thick skeleton from 3D porous objects while preserving the topological information. Models based on simplified skeletons have been shown to be efficient in retrieving morphological information from large scale disordered objects not only at a global level but also at a local level. In this paper, we present a series of 3D skeleton-based image processing techniques for evaluating the microarchitecture of large scale disordered porous media. The proposed skeleton method combines curve and surface thinning methods with the help of an enhanced shape classification algorithm. Results on two different porous objects demonstrate the ability of the proposed method to provide significant topological and morphological information.
\end{abstract}

\section{Keywords}

- Segmentation;

- Skeleton;

- Classification;

- Topological and morphological information;

- Porous media

\section{Introduction}

Various domains are concerned with the characterization of porous media. Natural materials (e.g. wood, stones, Fontainebleau sands, biological materials such as bones) and manmade materials (e.g. industrial foams, ceramics, electronic nanodevices) are examples of porous media from different application fields. These porous objects are usually considered not only in industry but also in research as multiphase materials composed of several elements arranged in space as a complex, sometimes messy, network. The problems of porous media have raised considerable interest among the scientific community as they cover a wide range of applications and scales: the exploration of underground entities at the microscopic scale in geology, and the examination of soil structures [18], the degradation of monuments [27], or the analysis of bones and the synthesis of industrial materials [8]. In most of these problems, 
the challenge is to study the physical behavior of these objects by characterizing their complex geometry, in order to improve and enhance their performance (glass or carbon fiber), to avoid (or limit) weathering or deterioration (stones, metal oxidation), and to understand or predict their behaviors (soils, rock reservoir, concrete, bones). This requires determining the main characteristics (morphology, texture, topology, etc.) of these porous media.

Furthermore, the prediction of properties (e.g. transport or mechanical properties) by models and simulations needs a realistic description of the phases constituting these porous materials.

An accurate and powerful non-destructive method to characterize the complex microstructure of porous materials is high resolution X-ray Computed Tomography (XCT). This technique has gained considerably in importance in recent years for the 3D-characterization of materials [6], [44], [26], [9] and [24]. This is notably due to continuous improvements in Xray tubes and XCT devices that have led to laboratory systems which can now achieve resolutions down to $1 \mu \mathrm{m}$ and even below depending on the materials and their sizes [25], [15], [31] and [48].

Starting from these images, the characterization of porous material has gradually switched from classical, and often destructive, exploration methods (BET, mercury porosimetry) to a non-invasive and increasingly precise science, namely 3D digital image processing. It is often necessary to measure from the image a quantity related to the physical property to be characterized. For example, transport phenomena and permeability in rocks can be studied through the distribution of the grains and the geometry of the pore phase [18]. In materials science, the anisotropy of a structure characterized by a Fabric tensor reflects the main poroelastic directions [17]. In the biomedical field, quantification and understanding of the distribution of the structure primitives of a trabecular bone are relevant for both diagnosis and treatment of bone disease. Instead of direct mechanical testing which is destructive and life threatening, the classification of these primitives helps in simulating these mechanical tests in order to estimate certain mechanical properties, such as stiffness. These mechanical properties are usually determined not only by their porosity, but also by the arrangement of trabeculae in the 3D space [4], [3] and [5].

The image processing methods proposed in the literature for the characterization of porous media can be classified in two main categories: global and local. Global methods, which are based on geometric models or use image averaging methods, allow the extraction of various approximated structural indices, such as the number of solid forms, spacing and orientation of the form primitives [10], [11] and [12]. These architectural parameters evaluated at a global level suffer from a priori hypotheses defined in the models and lack of precision, but are often sufficient to characterize the density, morphology or the anisotropy of porous network.

In this paper, however, we focus mainly on local approaches since they allow the measurement of quantitative indices of the structure by detecting the form primitives of the network, i.e. extraction of the internal structures such as beams and plates (e.g in the case of trabecular bones), which are fundamentally different, providing a set of new measures available directly from the images [16], [12] and [14]. Our aim is to demonstrate that using well chosen image processing procedures and an accurate skeleton, it is possible to access different morphological and topological features of huge data only from a simple and faithful representation of the original object.

In general, local-based techniques work locally on the 3D volume, and make few or no geometrical approximations by extracting useful information in each voxel or structure 
primitive. For example, the Hoshen-Kopelman percolation method [21] allows the isolation and characterization of the connected sets of the object, namely solid or porous clusters in the case of a porous media. It actually performs a local decomposition based on a neighborhood criterion. The numerical calculation of topological constants, such as Betti numbers or Poincare Euler characteristics (i.e. connectivity), is also based on this type of local counting process within a neighborhood [35]. All these algorithms are strictly deterministic and quantify properties of the media exactly. For example, characterization of the thicknesses of the structure primitives is based on the principle of the thickness map proposed by Hildebrand and Ruegsegger [20]. The methods of 3D skeletonization, thinning, medial axis [7] and the Voronoi distance map [22] are all methods enabling the decomposition of the media into primitives based on a simplified representation, while retaining the useful structural information. The segmentation of porous objects using these local techniques allows the measurement of many classical morphological and topological parameters, supplemented by new descriptors of structure, form and anisotropy.

In this work, the different phases of the porous media need first to be distinguished (segmentation step) prior to calculating characteristics (e.g. porosity, specific surface, Euler number) or simulating properties (e.g. conductivity, mechanical properties) on 3D images of different porous samples. Unfortunately, artifacts and/or noise often prevent segmentation of the 3D raw images [28]. As shown in the following, the segmentation of 3D images is specific to the application, imaging modality and nature of the object to be studied. Hence, the segmentation process is often preceded by an image analysis procedure (based on mathematical morphology tools and classical denoising filters in our case). After segmentation, a binarized media (represented by only two phases) is obtained. If the phase of interest is the pore phase (e.g. stones), it is assigned the value 255 , and the solid phase the value 0 (the dual image is chosen if the phase of interest is the solid phase (e.g. bones)). Mechanical properties and topological and morphological characteristics are usually analyzed using the solid phase of the porous objects, whereas other properties such as the transfer properties are determined using the porous phase. Once the zone of interest is well identified, quantitative analysis of the datasets becomes possible.

To assist in the local decomposition of porous media, simplified skeleton-based methods have been investigated [42], [37] and [33]. Shape information has been integrated directly into the models proposed to enhance the efficiency of such methods in terms of accuracy [36],

[7] and [29]. A skeleton is characterized by the following properties [45]: minimum thickness, homotopy equivalence, geometry preservation and reversibility.

Most variants of 3D binary skeletons are based on curve thinning or surface thinning. Since curve thinning does not preserve the geometry of non-cylindrical shapes, and surface thinning cannot erode beam shapes sufficiently, neither curve nor surface thinning is suitable for the analysis of porous media. To overcome this problem, shape information should be integrated into the skeleton models. A compound curve-surface (called here hybrid) skeleton has recently been proposed [2] and [23] to create structural models that take into account the shape of the object. These models enable a precise description of the local geometry.

In this paper, based on a previously proposed curve-surface thinning method [23], a complete image processing procedure is proposed to characterize different porous materials: the pore phase of limestone and solid phase of trabecular bones. Using different morphological and topological features we show that it is possible to distinguish between two sets of trabecular bones composed of osteoarthritic and osteoporotic samples. In addition, our proposed method 
is validated on geological applications (building stones) by the characterization of stone images.

The paper is organized as follows. Section 2 describes the materials and the methods used in this work. Section 3 presents the experimental results obtained on two different porous media (trabecular bone and limestones). Some concluding remarks are discussed in Section 4.

\section{Materials and methods}

To highlight the performance of the proposed method, two radically different porous media were chosen: limestones and human bones. The former are studied in the field of building conservation and the characterization of the porous phase is imperative to model water transport at the pore scale [38]. The latter are studied in the field of research against bone disease such as osteoporosis. The characterization of the solid phase is imperative to enhancing the model morphology, topology and mechanical properties.

\subsection{Stone sample description}

The limestones used in this study originate from a quarry in the north of Paris (SaintMaximin). Two beddings commercially denoted "Roche Franche" (RF) and "Roche Franche Fine" (RFF) were selected (Fig. 1(a)). These rocks are beige colored sedimentary limestones composed essentially of calcite and quartz with porosities of about $35 \%$ (RF) and $25 \%$ (RFF) and are of middle Lutecien age (i.e. formed 45 million years ago). These stones were widely used in the construction of monuments in Paris [39]. Five samples were selected from each stone type. The samples used in this study were rod samples drilled parallel to the stone bed.

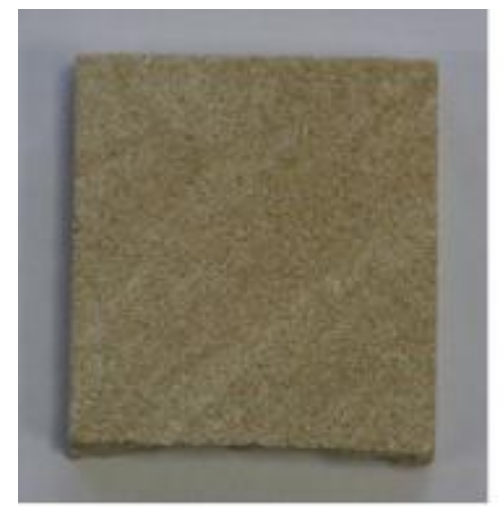

(a)

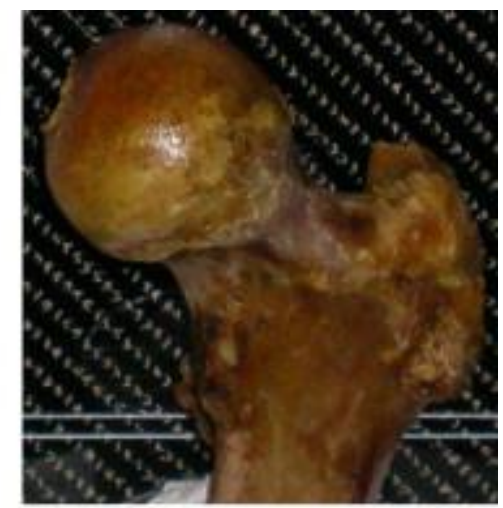

(b)

Fig. 1. : 3D porous objects: a limesstone (a) and a human femoral head (b).

\subsection{Bone sample description}

With the help of surgeons at the Hospital of Orleans in France, cylindrical samples were extracted from frozen human femoral heads (Fig. 1(b)) from patients with an a priori knowledge of fracture risk. In this study, we used five osteoarthritic (OA) samples and five osteoporotic (OP) samples. The bone structure of OA patients are known to be hypertrophied with an increased bone density, while the OP patients are characterized by the deterioration in the bone micro architecture which leads to bone fragility. They were prepared under 
continuous water irrigation using a precision diamond circular saw. The samples were oriented with respect to anatomic axes and cut to a dimension of $6 \mathrm{~mm}$ thickness and $8 \mathrm{~mm}$ diameter. All the samples were defatted chemically in order to conserve only the two main phases: trabeculae and pores.

\subsection{Acquisition of 3D images}

Briefly, a specimen was placed on a rotary stage between the X-ray source and the detector. The specimen was rotated step by step, taking one projection image at each angular position. Due to absorption by the material, the X-ray beam was attenuated when passing through a specimen. The change of intensity of the X-ray beam was recorded, resulting in gray level images (projections). Using a filtered back projection algorithm, a computer reconstructed the projections to obtain cross-sectional images of the sample (tomograms). Stacking these reconstructed images forms a 3D image of the sample (volume dataset). At each space position of the resulting dataset, a gray-value corresponds to the effective X-ray attenuation coefficient. Therefore, if the principal compounds of the object are known and have a sufficient density contrast, the distribution of these compounds within the object can be easily deduced. Theoretically, with a monochromatic X-ray (e.g. from a synchrotron beamline), the relation between pixel value (attenuation coefficient) and the compound's mass density is well defined by the Beer-Lambert equation [6]. With a polychromatic X-ray beam, which is the case for laboratory XCT, artifacts are inevitable and difficult to remove. It is therefore difficult to associate pixel values with material densities in an absolute and quantitative manner. Frequently encountered artifacts include: beam hardening effect [46] where an object of uniform density appears to have a thick and dense skin or ring effect [30] generally caused by temporary and spurious bad pixels in the detector. These effects can be reduced by the reconstruction software, but only to a certain extent and less effective for a multi-component object.

For the stone samples, microtomography analyses were performed using an industrial CT device Nanotom 180NF (GE Phoenix|X-ray, Wunstorf, Germany) available at the ISTO. This unit has a $180 \mathrm{kV}$ nanofocus X-ray tube and a digital detector array $(2304 \times 1152$ pixels Hamamatsu detector). Samples were placed in the chamber and rotated by 360 degrees during acquisition. The resulting projections were converted into a 3D image stack using a microcluster of four PCs with the Phoenix 3D reconstruction software (filtered back projection Feldkamp algorithm [19]). The reconstruction software contains several different modules for artifact reduction (e.g. beam hardening, ring artefacts) to optimize the results. Finally, the 16bit 3D image was converted into 8-bit (256 gray levels) for the image analysis processing. The samples (in the form of $6 \mathrm{~mm}$ diameter rods) were mounted and waxed on a glass rod. An operating voltage of $110 \mathrm{kV}$ and a filament current of $59 \mu \mathrm{A}$ were applied. The distance between the X-ray source and the sample and between the X-ray source and the detector was 15 and $500 \mathrm{~mm}$, respectively, giving a voxel size of $3 \mu \mathrm{m}$. The 2000 projection images (angular increment of $0.18^{\circ}$ ) were acquired during stone rotation (with an acquisition time of $4 \mathrm{~h}$ ). Using the central part of the 3D image, an isotropic region of interest of $256^{3}$ voxels was selected for both RF and RFF stone samples (Fig. 2). 


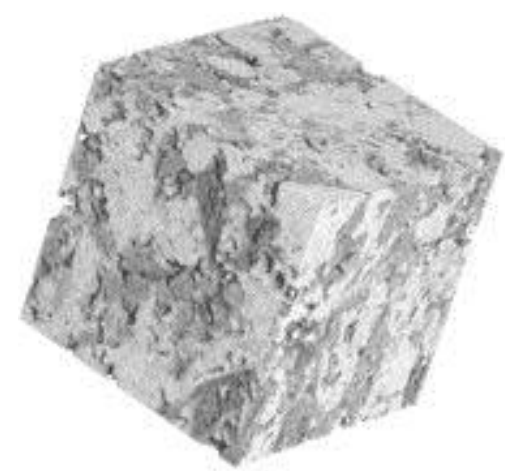

(a)

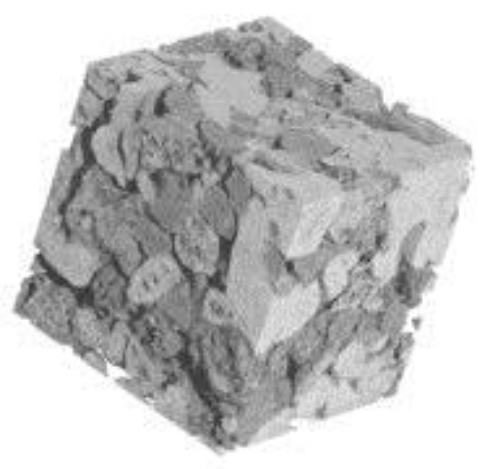

(b)

Fig. 2. : Area of interest of $256^{3}$ voxels selected from two different stone samples: RFF sample (a) and RF sample (b).

For the bone samples, images were obtained using the Skyscan 1072 high-resolution $\mu \mathrm{CT}$. The X-ray source was set at $80 \mathrm{kV}$ and $100 \mu \mathrm{A}$, and the magnification was set so as to obtain a pixel size of $12 \mu \mathrm{m}$. A $1024 \times 1024$ 12-bit digital cooled CCD coupled to a scintillator was used to record the radiographic projections. A total of 209 projections were acquired over an angular range of $180^{\circ}$ (angular step of $0.9^{\circ}$ ). Due to the cone beam, the radiographic images were processed with the Feldkamp algorithm. All the radiographic images were used to reconstruct the image slices using the Conebeam Reconstruction Software, version 2.6. Using the central part of the 3D image, an isotropic region of interest of $256^{3}$ voxels was selected for both OA and OP bone samples, as seen in Fig. 3.

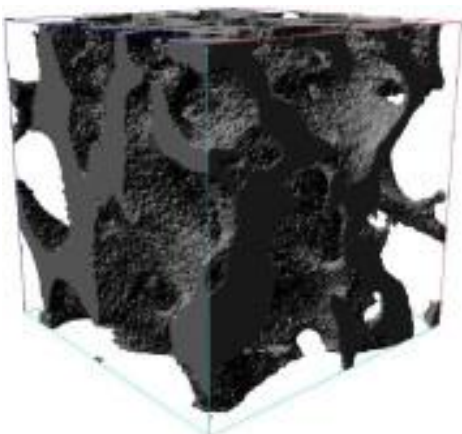

(a)

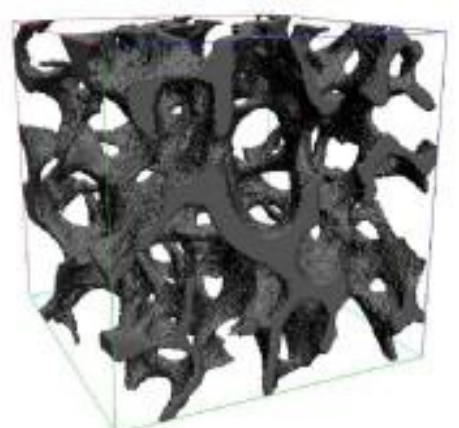

(b)

Fig. 3. : Area of interest of of $256^{3}$ voxels selected from two different bone samples: OA sample (a) and OP sample (b). Bone material is in gray color while pore material is in white.

\subsection{Segmentation of 3D images}

Since our image processing tools are only applicable to binarized images, the 3D raw data of bone and stone samples were pretreated (segmented). Segmentation is the process of partitioning the billions of gray level voxels of the $3 \mathrm{D}$ image into distinct phases. This is rarely a trivial and automatic process. 
The segmentation process applied on the two materials presented in this article consists of three main operations: filtering, binarization and correction. The following paragraphs describe each image processing operation.

\subsubsection{Filtering}

Using imaging systems, acquired real data are usually noisy, with a signal to noise ratio that depends on the quality of the acquisition equipment. If data are acquired from a laboratory $\mu \mathrm{CT}$ scanner, the noise might be linked to blurring, hardening of the beam, or its conical geometry, making the reconstruction difficult.

In the stone case, as previously mentioned, the gray level value of a voxel is related to the Xray absorption of the sample at the voxel position. Thus, in the case of the stone samples, pores appear in dark gray, silica compounds in medium gray and calcite compounds in light gray (Fig. 4). Even if these different phases are distinguishable to the naked eye, direct thresholding of the raw image is not possible. Indeed, the raw gray level histogram (Fig. 5) does not show any well defined peaks. Hence, most of the segmentation complexity is related to the presence of noise (voxels with the same gray value can in fact belong to two distinct phases) and blur (the borders between the phases are not well defined) [28]. To solve this problem, alternate sequential filters [28] were first used to remove the noise, but as they destroy the structural components smaller than the structuring element used, they cannot be pursued far enough.

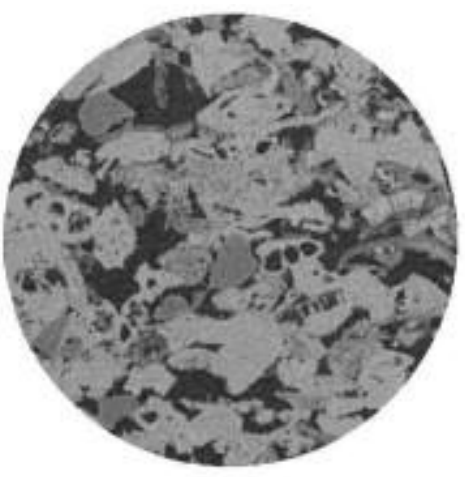

(a)

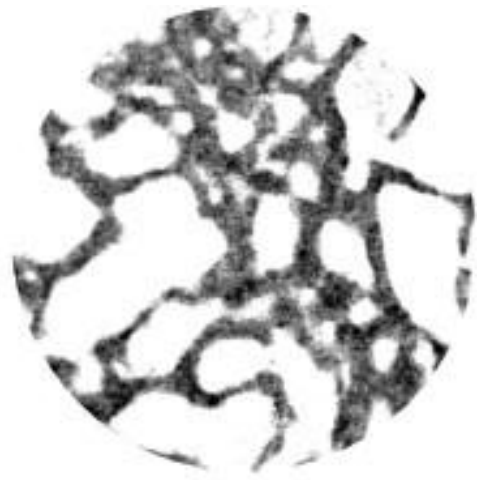

(b)

Fig. 4. : Selected 2D cuts of the stone (a) and bone (b) samples.

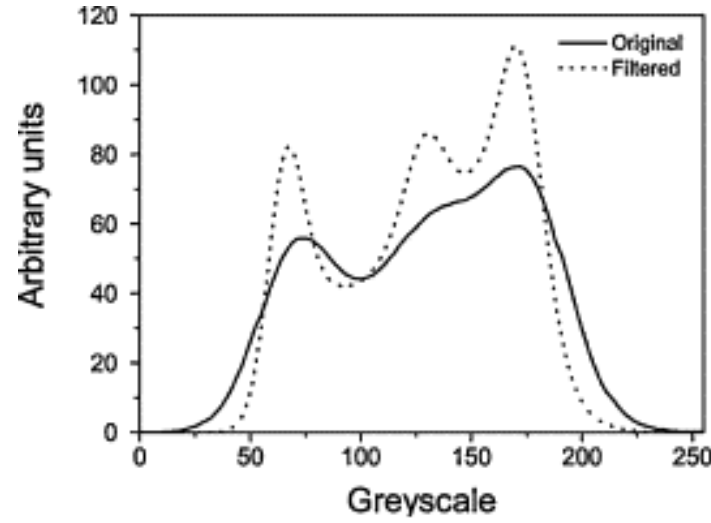

(a)

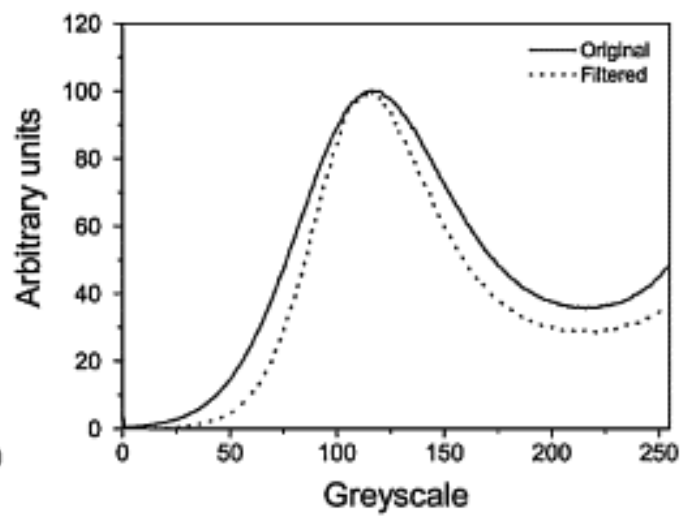

(b) 
Fig. 5. : Grayscale histograms recorded for a stone sample (a) and a bone sample (b), compared to the histogram of the filtered samples.

The stone samples were filtered using an erosion-dilation based method. In the following, a brief description of the method used is given. Let us define $\Psi$ as a $3 \mathrm{D}$ image consisting of a set of $N \times N \times N$ voxels, $N \in \mathbb{N}$, on a cubic grid with 26-neighboring voxels. For a 8-bit image, each voxel carries an integer value (gray level) in the range of $[0,255]$ :

$\Psi=\left\{v_{i, j, k}\right\}, v_{i, j, k} \in[0,255],(i, j, k) \in[0, N-1]$.

Each voxel is located in the image with a unique triplet of numbers (coordinates):

$\Psi(\vec{x})=v_{i, j}, k, \vec{x}=\{i, j, k\}$.

The erosion $\varepsilon$ and the dilation $\delta$ by a structuring element B for a gray level image are defined at every point $x$ by [43] :

$\delta_{B}((\Psi)(\vec{x})=\mathrm{V}\{\Psi(\vec{x}-\vec{y})\}, \vec{y} \in B(\vec{x})$, $\varepsilon B(\Psi)(\vec{x})=\Lambda\{\Psi(\vec{x}-\vec{y}),-\vec{y} \in B(\vec{x})\}$.

where $V$ is the supremum (or maximum) operator and $\Lambda$ the infinimum (or minimum) operator and $B(x)$ is the structuring element centred at the point $x$. The opening $\gamma$ and closing $\varphi$ are defined by the equations:

$\gamma_{B}=\delta B \varepsilon B$,

$\varphi B=\varepsilon B \delta B$,

A particular family of digital balls $B_{\lambda}$, with radius $\lambda$, were used for the structuring elements:

$B \lambda(\vec{x})=\{\vec{y}, d(\vec{x}, \vec{y}) \leq \lambda\}, \lambda \in \mathrm{N}$,

where $d(\vec{x}, \vec{y})$ is the Euclidean distance between the centers of the two voxels at coordinates

$\vec{x}$ and $\vec{y}$ in voxel size unit. These balls are a better approximation of the Euclidean sphere than those based on the digital distance $d_{26}$ and they do not penalize the efficiency of the implementation [28]. Sequential alternate filtering was applied up to a size $\lambda=3$, leading to the filtered image $\Psi_{f}$ :

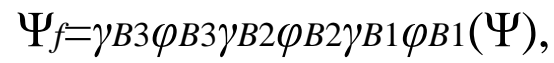

he digital ball $B_{3}$ has a diameter of 7 voxels $(7 \times 3=21 \mu \mathrm{m})$ which enables preserving the smallest elements of interest in the image. Improvement after denoising appears clearly on the histogram of the filtered image in Fig. 5(a). Indeed, it is difficult to distinguish the different stone phases from the histogram of the original (non-filtered) image. However, after filtering, three peaks are easily distinguished corresponding to the porous, calcite and silica phases of the stone. 
In the bone case, the task is less complicated. The first step consists in filtering the samples with a standard median $3 \times 3$ filter to reduce possible artifacts [40]. As can be seen from Fig. 5 (b), the histograms of the bone images before and after filtering are very close.

\subsubsection{Binarization}

In the bone case, this step aims at separating bone tissue from pore voxels. However, the distinction between the two phases in micro-scanner images is not always very intuitive as the borders separating the gray levels are not clear. Innovative techniques have been proposed to solve this problem [32] and [47]. We chose a standard binarization method using a threshold determined as the local minimum after the first mode of the histogram of each image [13]. If $\zeta$ is the binarization threshold, all voxels with a gray level higher than $\zeta$ are considered as solid (bone) $V_{\text {out }}=255$, otherwise, as void (pore) $\left(V_{\text {out }}=0\right)$, where $V_{\text {out }}$ is the gray values after the binarization operation.

In the stone case, and after the filtering step, three peaks are easily distinguished (Fig. 5(a)), which enables direct thresholding of the three phases. The thresholds are the gray values corresponding to the local minima of the histogram. Hence, all the voxels belonging (i) to the $\left[0, \zeta_{L}\right]$ range represent the pore phase, (ii) to the $\left[\zeta_{L}, \zeta_{H}\right]$ range represent the silica phase and (iii) to the $\left[\zeta_{H}, 255\right]$ range represent the calcite phase. As we are concerned by the water transfer in stones, the phase of interest is the pore phase. In other words, the value 255 is assigned to the porous phase (gray levels $\in\left[0, \zeta_{L}\right]$ ), and the value 0 is assigned to the solid phase (gray levels $\left.\in] \zeta_{L}, 255\right]$ ). In the case of the stone image (Fig. 5(a)) the threshold value used is $\zeta_{L}=96$.

\subsection{Correction}

After the binarization step and in order to take into account the anatomical constraints of porous objects, a correction step was applied. Each porous object treated in this study was considered as a binarized object that has one connected structure. This assumption is well justified for bone as well as for stone. In fact, bone tissues consist of one solid phase (all trabeculae are interconnected). In addition, the porosity of the stones is composed of open and closed porosities. In this study, all the enclosed pores were removed and only the open pores were kept (percolating cluster).

A technique based on Betti numbers was performed to ensure that the object consisted of only one connected structure. Considering a 3D space, there are 3 distinct Betti numbers that completely define the topology of an object. $\beta_{0}$ is the number of connected elements of the solid phase $\Omega$. $\beta_{1}$ is the number of loops and closed paths of $\Omega$. $\beta_{2}$ is the number of internal cavities of $\Omega$. The connectivity of an object is usually evaluated using the Euler-Poincare Characteristic, N3. It is linked to the Betti numbers through the following equation:

equation(9)

$N 3=\beta_{0}-\beta_{1}+\beta_{2}$

Based on Betti numbers, one connected component corresponds to $\beta_{0}=1$, and the number of cavities should be 0 which corresponds to $\beta_{2}=0$. Using the Hoshen-Kopelman (HK) algorithm [21], the connected clusters can be deterministically be separated. First, the HK algorithm was applied on the solid part and the principal cluster was kept, whereas the 
unconnected parts were removed. Then, the HK algorithm was applied on the porous part and the principal cavities were kept. All other small cavities were reassigned to the solid cluster to which they belong biologically. At the end of the correction step, the object should have only two phases: a 26-connected solid phase and a 6-connected pore phase.

The next section describes our spatial decomposition of the resulting one-component stone and bone structures (Fig. 6(a)) into rods and plates, for which the individual properties were associated.

(a)

Stone sample

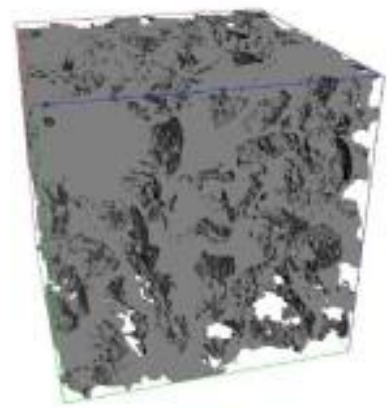

(b)

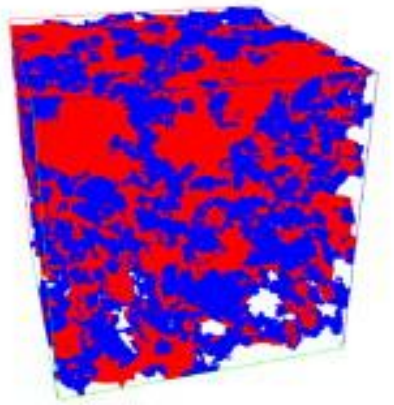

(c)

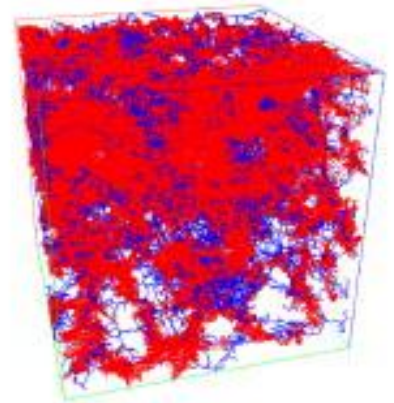

(d)

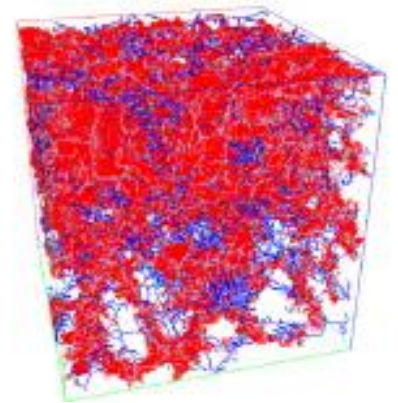

Bone Sample
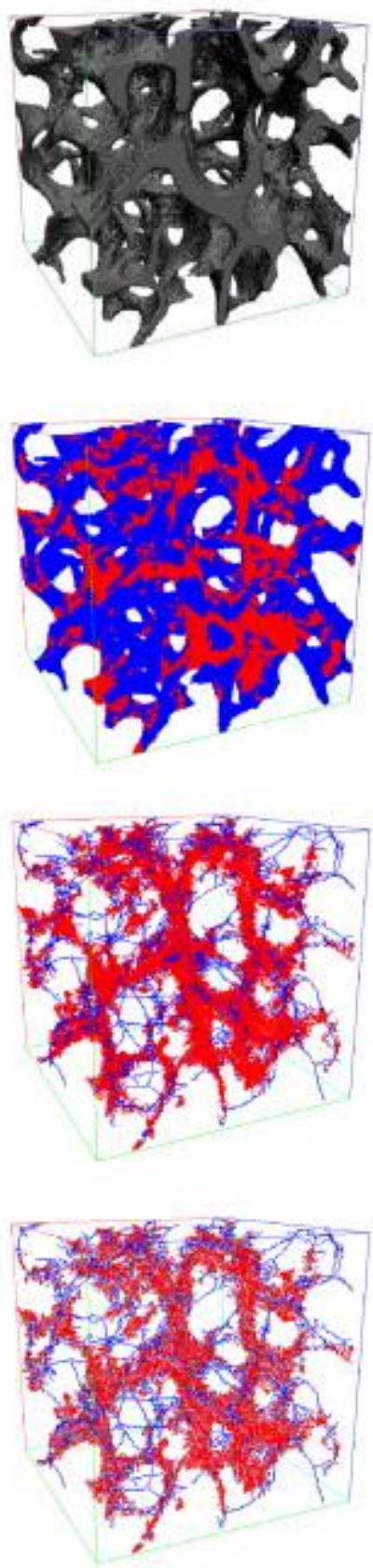

Fig. 6. : 3D porous media: one-connected objects (a), classified volumes, (b) hybrid skeletons, (c) and the final individualized skeletons (d). Rod voxels are in blue, plate voxels are in red while node and border voxels are in green. 


\subsection{Hybrid skeleton method}

Structural decomposition of skeleton structures has been investigated to characterize the morphological and topological properties of porous media. In [37], curve thinning was applied, representing each object's element by a 1D median path. However, this technique becomes less effective when the object's elements cannot be properly modeled by a cylinder, as in the case of stone and trabecular bone. Unlike curve thinning, surface thinning provides a good modeling of plate-like shapes, representing them by a 2D median surface. In surface thinning, which rarely generates a 1D-path skeleton, the rod forms are not well represented. To overcome these limitations, the object's structure needs to be classified as rod-like or platelike elements before being thinned. Surface thinning is therefore applied only on plateclassified elements, whereas curve thinning is applied on rod-classified elements

\subsubsection{Classification}

This step aims to classify each voxel of the skeleton as belonging to plate or rod elements. The objective is to guide the skeletonization process. Based on [7], a recently reported shape classification method [1] and [23] was used to decompose the object in two sets of plates and rods, taking into account the local shape of the porous object. This classification was performed at the voxel level. Each voxel, $m$ was assigned a rod, plate, border or node label, using a local topological analysis of the neighborhood voxels $N_{26}(m)$. The voxel classification function $f(m)$ can be expressed as follows:

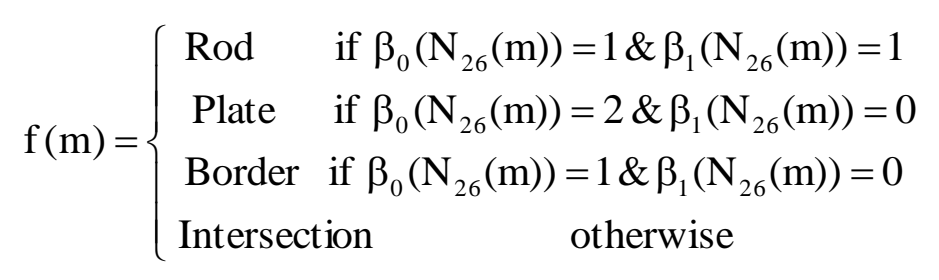

First, this function is applied on the surface skeleton generated from the original object. Then, a region growth step [23] is applied to propagate the labels to the entire volume. Finally, the resulting 4 classes are merged into 2 classes: rod and plate. In this merging process, iterative 6-connection propagations in the 6 directions of the space are performed. Each voxel labeled rod or plate attributes its label to its neighborhood in the considered direction, unless this neighborhood has already been assigned a rod or plate label. This process is iterated until no more than two classes (rods and plates) are found, as shown in Fig. 6(b). The order of the propagation in the 6 directions of the space determines which voxel has to be labeled and the error of the labeling step does not exceed one voxel.

\subsubsection{Skeletonization}

After the classification step, surface [34] and curve [33], thinning techniques were applied respectively to the plate and rod subsets. The joint use of these thinning techniques generated a skeleton composed of both 2D surfaces at plate shapes and 1D paths at rod shapes, as shown 
in Fig. 6(c). Using this approach, the resulting skeleton consists of a reasonable combination of curves and surfaces. The compound curve-surface skeleton is called here hybrid skeleton. The hybrid skeleton exploits the advantage of each technique by switching the conditions of the morphological erosion from one to another depending on the local form of the structure. Thus, the hybrid skeleton better preserves the topology of the original structure [2] and [23].

\subsubsection{Individualization}

The 3D skeleton was then individualized using a modified classification algorithm, originally proposed by Saha and Chaudhuri [41]. Instead of eight classes identified by the original algorithm, we propose to identify voxels in four classes, according to their structural roles: surface, curve, line-end (border) and node (intersection) voxels. This classification approach implements a "functional" rather than "discrete" geometry. The single class Node represents an interface zone between primitives of forms of whatsoever kind, and allows great freedom of modeling. Thus, we allow the management of various, sometimes complex, intersections avoiding the ambiguities that occur in the case of intersections of multiple surfaces and multiple curves. Fig. 6(d) represents the skeleton of the two porous materials after the individualization step.

It should be noted that after the classification step, each skeleton voxel is considered to correspond to either rod or plate element. However, after the individualization step, all voxels of each rod or plate are assigned a label which is different from those assigned to other rod or plate elements.

Note also that all parameters of the different stages, from segmentation to individualization of the proposed procedure are automatically initialized. The same procedure can be applied to any porous media. However, the segmentation step should be adapted according to the application in consideration.

\subsection{Morphological and topological features}

Interesting features characterizing the morphology and the topology of each sample can be extracted directly from the skeleton [23]. These features include: skeleton Euler Density (Euler.D), Skeleton density, skeleton volume (SV) to total volume (TV) or (SV/TV), normalized Number of Line-end voxels $($ Le.N), normalized Number of Node voxels $(N o . N)$ and Skeleton Curves to Skeleton Surfaces ratio $(S C / S S)$. Let us define $\Omega$ and $\Omega_{c}$ as the set of solid and pore voxels respectively, $T V$ as the total volume in $\mathrm{mm}^{3}, \rho$ as the voxel resolution in $\mathrm{mm}^{3}, \operatorname{Card}(\psi)$ as the number of elements in the set $\psi$, and $S_{H}$ as the set of voxels of the hybrid skeleton. $S_{H C}$ and $S_{H S}$ denote the curve part and the surface part of the hybrid skeleton respectively. $N_{26}(m)$ represents the 26-neighborhood of voxel $m$.

$$
\begin{aligned}
& \text { Euler.D }=\frac{\mathrm{N} 3}{\mathrm{TV}}=\frac{\beta_{0}-\beta_{1}+\beta_{2}}{\operatorname{Card}\left\{\Omega \cup \Omega_{\mathrm{c}}\right\} \times \rho} \\
& \frac{\mathrm{SV}}{\mathrm{TV}}=\frac{\operatorname{Card}\left\{\mathrm{S}_{\mathrm{H}}\right\}}{\operatorname{Card}\left\{\Omega \cup \Omega_{\mathrm{c}}\right\}}
\end{aligned}
$$




$$
\text { Le. } \mathrm{N}=\frac{\operatorname{Card}\left\{\mathrm{m} \in \mathrm{S}_{\mathrm{H}} \mid \beta_{0}\left(\mathrm{~N}_{26}(\mathrm{~m})\right)=1 \& \beta_{1}\left(\mathrm{~N}_{26}(\mathrm{~m})\right)=0\right\}}{\operatorname{Card}\left\{\Omega \cup \Omega_{\mathrm{c}}\right\}}
$$

$$
\text { No.N }=\frac{\operatorname{Card}\left\{m \in S_{H} \mid \beta_{0}\left(\mathrm{~N}_{26}(\mathrm{~m})\right)>\left(2-\beta_{1}\left(\mathrm{~N}_{26}(\mathrm{~m})\right)\right)\right\}}{\operatorname{Card}\left\{\Omega \cup \Omega_{\mathrm{c}}\right\}}
$$

$$
\frac{\mathrm{SC}}{\mathrm{SS}}=\frac{\operatorname{Card}\left\{\mathrm{S}_{\mathrm{H}_{\mathrm{C}}}\right\}}{\operatorname{Card}\left\{\mathrm{S}_{\mathrm{H}_{\mathrm{S}}}\right\}}
$$

The Euler.D is usually used to express the object connectivity. The SV/TV parameter reflects the presence of plates in the porous samples. The more plates there are in the sample, the higher the values obtained for the SVITV parameter. The number of line-ends $(L e . N)$ is an indicator of the number of broken branches. The Number of nodes $(N o . N)$ is considered as indices of element interconnection. The hybrid skeleton consists of a set of curves and surfaces. Two efficient and complementary features (number of curve and surface voxels) are consequently provided. In this study, these two values are represented as a ratio of Skeleton Curve voxels to Skeleton Surface voxels $(S C / S S)$.

\section{Results}

The results of Table 1 and Table 2 represent the values of the previously described parameters extracted from the hybrid skeleton of stone and trabecular bone samples, respectively. A bilateral hypothesis test was used to evaluate the discriminative power of each feature. Any parameter is said to separate the two sets of data if the Students $|t|$ value exceeds 3.35 for a high level of significance of 0.01 with a freedom degree of 8 . 
Table 1. : Mean \pm standard deviation and the Student $|t|$ values for different features estimated from 10 samples of porous stone objects. Any parameter is said to separate the two sets of data if $|t|$ value exceeds 3.35.

\begin{tabular}{|c|c|c|c|c|c|}
\hline \multirow[t]{2}{*}{ Feature name } & \multicolumn{2}{|c|}{ RF samples } & \multicolumn{2}{|c|}{ RFF samples } & \multirow[t]{2}{*}{ Student $|t|$ values } \\
\hline & $\mu$ & $\sigma$ & $\mu$ & $\sigma$ & \\
\hline $\mathrm{SV} / \mathrm{TV}\left(\times 10^{-3} \mathrm{~mm}^{-3}\right)$ & 24.19 & 1.91 & 4.86 & 2.35 & 12.77 \\
\hline Euler.D & -4050 & 624.1 & -571 & 235.40 & 10.43 \\
\hline Le. $\mathrm{N}\left(\times 10^{-3} \mathrm{~mm}^{-3}\right)$ & 0.31 & 0.01 & 0.08 & 0.04 & 11.16 \\
\hline No.N $\left(\times 10^{-3} \mathrm{~mm}^{-3}\right)$ & 5.55 & 0.42 & 1.61 & 0.75 & 9.17 \\
\hline $\mathrm{SC} / \mathrm{SS}$ & 0.16 & 0.01 & 0.36 & 0.05 & 7.84 \\
\hline
\end{tabular}

Table 2. : Mean \pm standard deviation and the Student $|t|$ values for different features estimated from 10 samples of trabecular bone data. Any parameter is said to separate the two sets of data if $|t|$ value exceeds 3.35.

\begin{tabular}{|c|c|c|c|c|c|}
\hline \multirow{2}{*}{ Feature name } & \multicolumn{4}{|c|}{ OA samples OP samples } & \multirow{2}{*}{ Student $|t|$ values } \\
\hline & $\mu$ & $\sigma$ & $\mu$ & $\sigma$ & \\
\hline $\mathrm{SV} / \mathrm{TV}\left(\times 10^{-3} \mathrm{~mm}^{-3}\right)$ & 24.97 & 6.82 & 10.52 & 0.87 & 4.20 \\
\hline Euler.D & -29.55 & 8.03 & -9.47 & 3.56 & 4.57 \\
\hline Le. $\mathrm{N}\left(\times 10^{-3} \mathrm{~mm}^{-3}\right)$ & 0.31 & 0.05 & 0.17 & 0.03 & 4.80 \\
\hline No.N $\left(\times 10^{-3} \mathrm{~mm}^{-3}\right)$ & 7.00 & 1.97 & 2.18 & 0.28 & 4.84 \\
\hline $\mathrm{SC} / \mathrm{SS}$ & 0.13 & 0.03 & 0.27 & 0.05 & 4.80 \\
\hline
\end{tabular}

As can be seen, in addition to globally measured parameters such as SVITV, Euler.D and $S C / S S$, the locally measured parameters such as Le.N and No.N allow the distinction between the OA and OP samples in the case of bone, and between the two RF and RFF sets in the case of stone. Furthermore, the values within each group of five samples are very close, providing an additional measure of similarity. The skeletons of osteoporosis bone tend to have fewer line-end voxels. Similarly, the skeletons of RFF (fine stones) provide a Le.N that is slightly less than those provided by the RF stone samples. It should also be noted that $N o . N$ can be considered as a good discriminator parameter as the values obtained for the trabecular bone or stone samples are very different. As shown in Table 1, the RF sample is more connected (higher Euler.D and No.N numbers), with bigger voids (higher SVITV numbers) and with higher dead-ends (interesting for dissolution-recrystallization processes) than for the RFF sample. 


\section{Conclusion}

In this paper, a complete image processing (IP) procedure based on curve-surface skeleton is proposed and presented as a powerful tool for the analysis of disordered porous media. The proposed IP procedure is based on the evaluation of the local shape of the structure primitives. In fact, each plate-like and rod-like element is distinguished. Consequently, precise features in terms of microarchitecture can be estimated. Based on real porous data, the proposed method was able to better distinguish between different samples.

Different features linked to the morphology and the topology of the studied data were proposed. The results obtained on the different samples show that these features enable the different samples to be distinguished. The drawback of this study is the sample size. We are currently investigating the use of the proposed method for the characterization of a more complete database. Moreover, an appropriate classification method will be considered to determine how to combine the different features for a better discrimination of the studied samples. In addition to morphological analysis, the new model can be used as a basis for mechanical studies using finite elements (FE). The long-term aim of our work is to develop a biomechanical simulation protocol that could be used to virtually characterize the stiffness of porous media such as bones and stones. This would contribute significantly to detecting bone fragilities simply by acquiring 3D images, for example with a high-resolution in-vivo CT, and simulating mechanical compression using fast and precise FE models, where the precise geometrical description of the medium is essential. Based on the separation of rod-like from plate-like shapes, the thinning and individualization methods proposed in this paper are currently under investigation, with the objective of improving the geometrical deficiency of simplified FE models and reducing the complexity of the micro-mechanical analysis of porous structures.

\section{References}

G. Aufort, R. Jennane, R. Harba, C.L. Benhamou

Hybrid skeleton graph analysis of disordered porous media. application to trabecular bone

Proceedings of the IEEE International Conference on Acoustics, Speech, and Signal Processing 2007, Toulouse, France (2006)

\section{G. Aufort, R. Jennane, R. Harba, C.L. Benhamou}

A new shape-dependant skeletonization method. application to porous media Proceedings of EUSIPCO 2006, Florence, Italy (2006)

\section{G. Aufort, R. Jennane, R. Harba, C.L. Benhamou}

Shape classification techniques for discrete $3 \mathrm{D}$ porous media. application to trabecular bone

IEEE International Conference on Engineering in Medicine and Biology Society, Lyon, France (2007)

G. Aufort, R. Jennane, R. Harba, A. Gasser, D. Soulat, C.L. Benhamou Nouvelle approche de modélisation de milieux poreux: Application à l'os trabéculaire

GRETSI'05, Louvain-la-Neuve, Belgium (2005), pp. 429-432 
Mechanical assessment of porous media using hybrid skeleton graph analysis and finite elements. application to trabecular bone

Proceedings of EUSIPCO 2007, Poznan, Poland (2007)

J. Baruchel, J.Y. Buffiere, E. Maire, P. Merle, G. Peix

$\mathrm{X}$-Ray tomomography in material science

Hermes Science (2000)

A. Bonnassie, F. Peyrin, D. Attali

A new method for analyzing local shape in three-dimensional images based on medial axis transformation

IEEE Transactions on Systems, Man and Cybernetics, 44 (4) (2003), pp. 700-705

J.P. Bonnet, F. Topin, L. Tadrist

Flow laws in metal foams: Compressibility and pore size effects

Transport in Porous Media, 73 (2) (2008), pp. 233-254

N. Burlion, D. Bernard, D. Chen

$\mathrm{X}$-ray microtomography: Application to microstructure analysis of a cementitious material during leaching process

Cement and Concrete Research, 36 (2) (2006), pp. 346-357

F. Cassot, P. Paulin-Laurens, F. Plouraboué, H. Duvernoy, J.-P. Marc-Vergnes

Morphometric study of a human cerebralmicrovascular network

Journal of Cerebral Blood Flow and Metabolism, 19 (suppl. 1) (1999) S708 21-37

F. Chaari, E. Markiewicz, P. Drazetic

Identification of the spongy bone mechanical behaviour under compression loads: numerical simulation versus experimental results

International Journal of Crashworthiness, 12 (3) (2007), pp. 247-253

C. Chappard, A. Basillais, C.L. Benhamou, A. Bonassie, B. Brunet-Imbault, N. Bonnet, F. Peyrin

Comparison of synchrotron radiation and conventional X-ray microcomputed tomography for assessing trabecular bone microarchitecture of human femoral heads

Medical Physics, 33 (9) (2006), pp. 3568-3577

C. Chappard, A. Marchadier, C.L. Benhamou

Side-to-side and within-side variability of 3D bone microarchitecture by conventional microcomputed tomography of paired iliac crest biopsies

Bone, 43 (1) (2008), pp. 203-208

C. Chappard, F. Peyrin, A. Bonnassie, G. Lemineur, B. Brunet-Imbault, E. Lespessailles, C.L. Benhamou

Subchondral bone micro-architectural changes in osteoarthritis: a synchrotron micro-computed tomography study

Osteoarthritis and Cartilage, 14 (3) (2006), pp. 215-223

V. Cnudde, J.P. Cnudde, C. Dupuis, P.J.S. Jacobs 
X-ray micro-ct used for the localization of water repellents and consolidants inside natural building stones

Materials Characterization, 53 (2004), pp. 259-271

Consensus, Development conference on osteoporosis, American Journal of Medicine 30 (95-5A) (1993) 1-78.

\section{S.C. Cowin}

Anisotropic poroelasticity: fabric tensor formulation

Mechanics of Cellular and Porous Materials, 36 (8) (2004), pp. 665-677

J.-F. Delerue, E. Perrier, A. Timmerman, R. Swennen

3D soil image characterization applied to hydraulic properties computation

F. Mees, R. Swennen, M. Van Geet, P. Jacobs (Eds.), Applications of X-ray computed tomography in the geosciences, 215The Geological Society of London, Special Publications (2003), pp. 167-176

L.A. Feldkamp, L.C. Davis, J.W. Kress

Box-skeletons of discrete solids

Journal of the Optical Society of America A, 1 (1984), pp. 612-619

T. Hildebrand, P. Ruegsegger

A new method for the model independent assessment of thickness in threedimensional images

Journal of Microscopy, 185 (1997), pp. 65-67

J. Hoshen, R. Kopelman

Percolation and cluster distribution. i. cluster multiple labeling technique and critical concentration algorithm

Physical Review B, 14 (1976), pp. 3438-3445

S. Impedovo, R. Modugno, G. Pirlo

Analysis of membership functions for voronoi-based classification

International Conference on Frontiers in Handwriting Recognition (ICFHR),

Kolkata, India (2010)

R. Jennane, A. Almhdie, G. Aufort, E. Lespessailles

3D shape-dependent thinning method for trabecular bone characterization

Medical Physics, 39 (1) (2012), pp. 168-178

A. Kaestner, E. Lehmann, M. Stampanoni

Imaging and image processing in porous media research

Advances in Water Resources, 31 (2008), pp. 1174-1187

R.A. Ketcham, W.D. Carlson

Acquisition and optimization and interpretation of $\mathrm{x}$-ray computed tomographic imagery: applications to the geosciences

Computers and Geosciences, 27 (2001), pp. 381-400

J. Lambert, I. Cantat, R. Delannay, A. Renault, F. Graner, J.A. Glazier, I. Veretennikov, P. Cloetens 
Extraction of relevant physical parameters from 3D images, of foams obtained by X-ray tomography

Colloids and Surfaces A: Physicochemical and Engineering Aspects, 31 (2005), pp. 295-302

\section{E. Le Trong, Y. Anguy}

Simplification d'images 3D de matériaux poreux en vue de leur caractérisation physique

Revue Européenne de Génie Civil, 9 (3) (2005), pp. 353-372

E. Le Trong, O. Rozenbaum, J.L. Rouet, A. Bruand

A simple methodology to segment X-ray tomographic images of a multiphasic building stone

Image Analysis and Stereology, 27 (2008), pp. 175-182

X. Liu, P. Saha, F. Wehrli, P. Sajda, X. Guo

A 3D morphological analysis of trabecular bone based on individual trabeculae segmentation

Transactions of the Orthopaedic Research Society, 31 (2006), p. 1783

A. Lumpkin, B. Yang, C. Yao, L. Emery

$\mathrm{X}$-ray imaging of the aps storage ring beam stability effects: from the alaskan earthquake to undulator field changes

Medical Imaging, 4 (2003), pp. 2423-2425

E. Maire, P. Colombo, J. Adrien, L. Babout, L. Biasetto

Characterization of the morphology of cellular ceramics by $3 \mathrm{~d}$ image processing of X-ray tomography

Journal of the European Ceramic Society, 27 (2007), pp. 1973-1981

S. Majumdar, H.K. Genant, S. Genant, D.C. Newitt, V.H. Truong, J.C. Lin, A. Mathur Correlation of trabecular bone structure with age, bone mineral density, and osteoporotic status: in vivo studies in the distal radius using high resolution magnetic resonance imaging

Journal of Bone and Mineral Research, 12 (1) (1997), pp. 111-118

D. G. Morgenthaler, Three-dimensional simple points: serial erosion, parallel thinning and skeletonization, Tech. Rep. TR-1005.

J. Mukherjee, P.P. Das, B.N. Chatterji

On connectivity issues of espta

Pattern Recognition Letters, 11 (9) (1990), pp. 643-648

A. Odgaard, H.J.G. Gundersen

Quantification of connectivity in cancellous bone, with special emphasis on 3D reconstructions

Bone, 14 (2) (1993), pp. 173-182

L. Pothuaud, A. Laib, P. Levitz, C.L. Benhamou, S. Majumdar 
Three-dimensional-line skeleton graph analysis of high-resolution magnetic resonance images: a validation study from 34-microm-resolution microcomputed tomography

Journal of Bone and Mineral Research, 17 (10) (2002), pp. 1883-1895

L. Pothuaud, P. Orion, E. Lespessailles, C.L. Benhamou, P. Levitz

A new method for three-dimensional skeleton graph analysis of porous media: application to trabecular bone microarchitecture

Journal of microscopy, 199 (2) (2000), pp. 149-161

O. Rozenbaum

3D characterization of weathered building limestones by high resolution synchrotron X-ray microtomography

Science of the Total Environment, 409 (10) (2011), pp. 1959-1966

O. Rozenbaum, L. Barbanson, F. Muller, A. Bruand

Significance of a combined approach for replacement stones in the heritage buildings conservation frame

Comptes Rendus Geoscience, 340 (2008), pp. 345-355

H. Sadoghi Yazdi, F. Homayouni

Impulsive noise suppression of images using adaptivemedian filter, International Journal of Signal Processing, Image Processing and Pattern Recognition, 3 (3) (2010), pp. 1-12

P.K. Saha, B.B. Chaudhuri

3D digital topology under binary transformation with applications

Computer Vision and Image Understanding, 63 (1996), pp. 418-429

P.K. Saha, B.B. Chaudhuri, D.D. Majumder

A new shape preserving parallel thinning algorithm for 3D digital images

Pattern Recognition, 30 (1997), pp. 1939-1955

J. Serra

Image Analysis and Mathematical Morphology, vol. IAc. Press, London (1982)

M. Stampanoni, G. Borchert, P. Wyss, R. Abela, B. Patterson, S. Hunt, D. Vermeulen, P. Ruegsegger

High resolution X-ray detector for synchrotron-based microtomography

Nuclear Instruments and Methods in Physics Research A, 491 (2002), pp. 291-301

A. Sudhalkar, L. Gursoz, F. Prinz

Box-skeletons of discrete solids

Computer-Aided Design, 28 (1996), pp. 507-517

E. Van de Casteele, D. Van Dyck, J. Sijbers, E. Raman

The effect of beam hardening on resolution in X-ray microtomography

Medical Imaging, 5370 (2004), pp. 2089-2096

B. Vasilic, F.W. Wehrli 
A novel local thresholding algorithm for trabecular bone volume fraction mapping in the limited spatial resolution regime of in vivo mri

IEEE Transactions on Medical Imaging, 24 (12) (2005), pp. 1574-1585

E. Vergès, D. Tost, D. Ayala, E. Ramos, S. Grau

$3 \mathrm{D}$ pore analysis of sedimentary rocks

Sedimentary Geology, 234 (2011), pp. 109-115 